IdeAs

Idées d'Amériques

$10 \mid 2017$

États-Unis / Cuba : une nouvelle donne?

\title{
Arnauld De Sartre, Xavier. Agriculture et changements globaux. Expertises globales et situations locales
}

Bruxelles. Peter Lang, 2016, 204 pages.

Solenn Le Clech

\section{OpenEdition}

Journals

Édition électronique

URL : https://journals.openedition.org/ideas/2104

DOI : $10.4000 /$ ideas. 2104

ISSN : $1950-570$

Éditeur

Institut des Amériques

Référence électronique

Solenn Le Clech, « Arnauld De Sartre, Xavier. Agriculture et changements globaux. Expertises globales et situations locales », IdeAs [En ligne], 10 | 2017, mis en ligne le 17 novembre 2017, consulté le 18 octobre 2022. URL : http://journals.openedition.org/ideas/2104; DOI : https://doi.org/10.4000/ideas. 2104

Ce document a été généré automatiquement le 18 octobre 2022

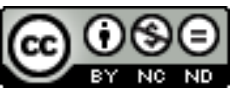

Creative Commons - Attribution - Pas d'Utilisation Commerciale - Pas de Modification 4.0 International - CC BY-NC-ND 4.0

https://creativecommons.org/licenses/by-nc-nd/4.0/ 


\title{
Arnauld De Sartre, Xavier. Agriculture et changements globaux. Expertises globales et situations locales
}

Bruxelles. Peter Lang, 2016, 204 pages.

\author{
Solenn Le Clech
}

\section{RÉFÉRENCE}

Agriculture et changements globaux. Expertises globales et situations locales, Bruxelles. Peter Lang, 2016, 204 pages.

1 L'Anthropocène est caractérisé par des changements globaux qui remettent en question notre société contemporaine et dont les impacts nécessitent une prise en charge politique. S'appuyant sur des cas d'étude contemporains en milieu agricole, l'ouvrage de Xavier Arnauld de Sartre propose une lecture ancrée dans la Political Ecology des grandes options envisagées pour permettre de faire face aux enjeux de l'Anthropocène. Ce faisant, il s'appuie sur un mélange subtil de considérations conceptuelles, cas concrets d'étude et expériences de terrain.

2 L'ouvrage peut se diviser en deux grandes parties. L'introduction et les deux premiers chapitres guident le lecteur dans une explication de la situation environnementale vue au prisme de la modernité. L'auteur propose ainsi une analyse critique des récits modernes en décrivant les enjeux de la modernité, ses grandes phases et ses tensions. Cette première partie donne des clés de lecture conceptuelles pour analyser les situations étudiées dans les trois chapitres suivants de l'ouvrage. Ceux-ci mobilisent chacun un terrain d'étude où coexistent des types d'agriculture ou de politique de gestion des espaces naturels très différents. Ces cas d'étude sont replacés dans un débat plus large opposant land sharing / land sparing et sont mis en perspective des scénarios du Millenium Ecosystem Assessment (MEA). 
3 Le chapitre 1 pose les bases conceptuelles de l'ouvrage en contextualisant les politiques environnementales à l'échelle globale à travers une réflexion sur les transformations de la modernité et l'institutionnalisation de ses critiques au sein de la modernité réflexive. Toutefois, $\mathrm{X}$. Arnauld de Sartre souligne l'insuffisance de cette dernière limitant ainsi l'adéquation des actions des politiques publiques. La crise de croyance dans les valeurs de la modernité, associée à des crises environnementale et économique, a donné lieu à l'institutionnalisation de solutions, dont les quatre scénarios du MEA qui sont repris dans la suite de l'ouvrage. Ces scénarios offrent des grilles de lecture et une repolitisation du futur en rappelant que celui-ci est une question de choix et leur application à l'agriculture permet d'analyser les solutions envisagées et la manière dont elles le sont.

4 Le chapitre 2 recentre le débat sur l'agriculture et rappelle l'injonction paradoxale à laquelle elle est confrontée : produire de la nourriture dans un contexte de croissance démographique tout en limitant les impacts négatifs sur l'environnement. Trois grands débats autour de l'agriculture se sont construits autour de ce paradoxe : la critique du modèle productif de la Révolution verte et la recherche de modèles alternatifs, la recherche de scénarios pensant le verdissement des formes d'agriculture et le débat land sparing/land sharing. Ces débats reposent la question de la prise en compte de la dimension spatiale puisque la complémentarité des espaces et la prise en compte de la diversité des situations constituent des éléments clé dans la recherche d'un équilibre global entre espaces de production et de préservation de l'environnement.

5 Le chapitre 3 se base sur l'exemple du système agroexportateur de la Pampa argentine où s'est mis en place un contexte favorable à la diffusion hégémonique du land sparing. Ce cas d'étude constitue un exemple paradigmatique de la modernisation écologique appliquée par l'intensification agricole et peut être considéré au travers du scénario Technogarden proposé par le MEA. L'intensification agricole s'est effectuée au cours d'une ébauche de troisième révolution agricole basée sur les nouvelles technologies et marquée par des transformations profondes sociétales, économiques, territoriales et techniques. Localement, l'intensification agricole et la diffusion de l'innovation reposent sur le tissu social et la structure foncière. A une échelle plus large, elles ont été rendues possibles par le désengagement de l'Etat qui a quasiment déserté le champ des politiques agricoles et mis le secteur agricole au contact direct des marchés internationaux. Le système agricole résultant est ainsi dépendant du système politique et du tissu social qui à la fois lui a donné naissance et l'a diffusé. Les problèmes économiques et écologiques qui émergent progressivement montrent les limites du modèle, voire le remettent en question.

6 Le chapitre 4 illustre un cas de choix de land sparing à partir de l'exemple du Gabon qui se situe entre les deux scénarios Ordre par la force et Orchestration globale du MEA. La division territoriale entre l'exploitation forestière et l'implantation de parcs nationaux est l'occasion de rappeler le rôle des politiques et ainsi de souligner l'importance de la compréhension des rapports de forces entre les acteurs d'un territoire. Le Gabon est souvent considéré comme un cas exemplaire de conservation des milieux. Celle-ci s'est effectuée dans le cadre d'une domination politique marquée par l'association d'un Etat rentier, fort et autoritaire et de deux ONG internationales dans un contexte postcolonial fort. Les populations locales sont exclues des parcs, sans recevoir de compensation, et sont de ce fait hostiles à leur implantation. L'inadaptation des politiques aux populations locales crée, et a déjà créé par le passé, de larges limitations 
qui peuvent conduire à l'abandon de projets. De ce fait, de nouveaux projets menés par une politique volontariste de l'Etat tentent de pallier ce problème en impliquant davantage les populations et en leur proposant des projets de développement.

7 Le chapitre 5 se penche sur le cas du Brésil, Etat fort et moderne qui a voulu assoir sa souveraineté territoriale sur le Bassin amazonien. Ce faisant, il s'est tourné vers le choix d'une articulation entre land sharing et land sparing, à l'articulation entre les scénarios Technogarden et Mosaïque adaptative. L'Amazonie brésilienne est soumise à de fortes pressions entre besoins alimentaires et protection environnementale. La solution actuelle proposée par un Etat volontariste se base à la fois sur la préservation relative de l'Amazonie compensée par une intensification agricole dans les Cerrados (land sparing) et sur le zonage de l'espace amazonien où des politiques environnementales et des modes de gestions différents s'appliquent (land sharing). Mené avec succès par les agriculteurs familiaux, le projet de land sharing est basé sur la recherche de modèles alternatifs, proches de la Mosaïque adaptative, qui découlent en systèmes souvent qualifiés d'agroécologie. Toutefois, Xavier Arnauld de Sartre présente les limites du modèle liées notamment au caractère éphémère du volontarisme de l'Etat, au manque de perspectives sur le long terme ou encore au manque de prise en compte des dynamiques régionales dans l'application locale de la politique de land sharing, en s'appuyant sur des cas d'étude locaux concrets.

La conclusion de l'ouvrage replace les cas d'étude dans le modernisme, par le rôle que joue l'Etat, la manière dont la nature est prise en compte et leur insertion dans le capitalisme. Confrontées à un besoin de changement, ces trois situations prennent chacune leur sens dans une déclinaison particulière de la modernité. Elles font face à des problèmes qui incluent les dynamiques héritées du modernisme : contestation des parcs, absence de modèle économique, mise à l'écart de la Nature et des populations locales et l'absence de compensations face à l'émergence d'un système durable. Malgré la nécessité de replacer les populations locales au cœur des défis portés par les transformations, les acteurs doivent développer des stratégies dans des figures spatiales héritées ou imposées, rendant difficile la mise en place de solutions alternatives. Le verrouillage de ces problèmes, économiques, spatiaux et/ou technologiques, explique ainsi les décalages entre enjeux et la situation actuelle.

\section{AUTEURS}

\section{SOLENN LE CLECH}

Postdoctoral fellow, Agricultural Economics and Policy (AECP) group, ETH Zürich, Switzerland. LETG Rennes, University Rennes 2, France 